\title{
ON PARACOMPACT SPACES ${ }^{1}$
}

CHUNG-TAO YANG

1. Stone ${ }^{2}$ has proved that a space is fully normal $T_{1}$ if and only if it is paracompact $T_{2}$. If throughout his proof $T_{1}$ is deleted and $T_{2}$ is replaced by $T_{4}$ (normality), we obtain that a space is fully normal if and only if it is paracompact $T_{4}$. In this note we prove that $T_{2}$ can also be replaced by any one of the following:

$T_{2}^{\prime}$. Every two points with disjoint closures have disjoint neighborhoods.

$T_{3}^{\prime}$. For each point $x$ and neighborhood $U$ of $\bar{x}$ there is a neighborhood $V$ of $x$ whose closure is contained in $U$.

$L T_{4}$. Every point has a neighborhood whose closure is normal.

Moreover, we shall also study a natural decomposition of a space with certain properties and prove that a space is paracompact $T_{4}$ if and only if it has a retract which is paracompact $T_{2}$ and meets every non-null closed set.

The following terminology will be used.

PROPERTy *. If $x, y, z$ are any three points such that $\bar{x} \cap \bar{y} \neq \varnothing$ and $\bar{x} \cap \bar{z} \neq \varnothing$, then $\bar{y} \cap \bar{z} \neq \varnothing$.

Property **. Every point has a compact closure.

We remark that

$$
\begin{aligned}
& T_{2} \rightarrow T_{2}^{\prime} \text { and } T_{3} \rightarrow T_{3}^{\prime} . \\
& T_{4} \rightarrow L T_{4} \rightarrow T_{3}^{\prime} \rightarrow T_{2}^{\prime} \rightarrow \text { property }{ }^{*} .
\end{aligned}
$$

2. Theorem 1. In a paracompact space, $T_{2}^{\prime}, T_{3}^{\prime}, L T_{4}$ and $T_{4}$ are equivalent.

Proof. It is immediate that $T_{4}$ implies $T_{2}^{\prime}, T_{3}^{\prime}$, and $L T_{4}$. To prove that $T_{2}^{\prime}$ plus paracompactness implies $T_{3}^{\prime}$ and that $T_{3}^{\prime}$ plus paracompactness implies $T_{4}$ we can simply follow Dieudonné's proof ${ }^{3}$ with $T_{2}$ and $T_{3}$ replaced by $T_{2}^{\prime}$ and $T_{3}^{\prime}$ respectively. For any paracompact $L T_{4}$ space there is a locally finite open covering, say $\left\{U_{\alpha}\right\}$, such that each $\bar{U}_{\alpha}$ is normal. Since $\left\{\bar{U}_{\alpha}\right\}$ is still locally finite, the un-

Received by the editors October 23, 1952 and, in revised form, August 11, 1953.

${ }^{1}$ One part of this work was done under Contract N7-434 Task Order III, Naval Department, Office of Naval Research.

${ }^{2}$ Cf. A. H. Stone, Paracompactness and product spaces, Bull. Amer. Math. Soc. vol. 54 (1948) pp. 977-982.

${ }^{3}$ Cf. J. Dieudonné, Une généralisation des espaces compacts, J. Math. Pures Appl. (9) vol. 23 (1944) pp. 65-76. 
proved part of Theorem 1 follows from the following more general result.

Lemma. ${ }^{4} A$ space which is covered by a locally finite system of normal closed sets is normal.

Proof. Let $E, F$ be disjoint closed sets in a topological space $X$ covered by a locally finite system of closed normal sets $A_{\alpha}$. For each $\alpha$, there exist, by the normality of $A_{\alpha}$, open sets $U_{\alpha}, V_{\alpha}$ in $X$ such that

$$
E \cap A_{\alpha} \subset U_{\alpha}, \quad F \cap A_{\alpha} \subset V_{\alpha} \text {, and } U_{\alpha} \cap V_{\alpha} \cap A_{\alpha}=\varnothing .
$$

Now we define

$$
\begin{aligned}
& P(x)=X-\cup\left\{A_{\alpha}: A_{\alpha} \boxplus x\right\}, \quad x \in X \text {; } \\
& Q(x)=\cap\left\{U_{\alpha}: A_{\alpha} \ni x\right\}, \quad x \in E \text {; } \\
& R(x)=\bigcap\left\{V_{\alpha}: A_{\alpha} \ni x\right\}, \quad x \in F .
\end{aligned}
$$

It follows by the local finiteness of the system $\left\{A_{\alpha}\right\}$ that $P(x), Q(x)$, and $R(x)$ are open. Let

$$
U=U\{P(x) \cap Q(x): x \in E\}, \quad V=U\{P(x) \cap R(x): x \in F\} .
$$

Then $U, V$ are disjoint neighborhoods of $E, F$.

REMARK. We can show by examples that in a pointwise paracompact space, $T_{2}^{\prime}, T_{3}^{\prime}, L T_{4}$, and $T_{4}$ are not equivalent to one another.

COROLlaRy. ${ }^{5}$ The product of a paracompact $T_{4}$ space and a compact $T_{4}$ space is paracompact $T_{4}$.

To prove this corollary we have only to observe that the product of a paracompact space and a compact space is paracompact ${ }^{6}$ and that the product of two $T_{2}^{\prime}$ spaces is $T_{2}^{\prime}$.

3. Given any space $X$ with property * we can define an equivalence relation such that two points $x, y$ of $X$ are equivalent if and only if $\bar{x} \cap \bar{y} \neq \varnothing$. This relation yields a decomposition $D$ of $X$, that is, a system of sets, pairwise disjoint, whose union is $X$ and such that two points of $X$ are contained in a same member of $D$ if and only if they are equivalent. Denote by $\phi$ the projection of $X$ onto $D$, i.e., the function of $X$ into $D$ such that $f(x)=p$ if $x \in p$. There is a topology on $D$ such that a subset $G$ of $D$ is open if and only if $\phi^{-1}(G)$ is open. $D$ with this topology is the natural quotient space of $X$. If $X$ is $T_{4}$ and then

4 This is suggested by the referee as a known but unpublished result.

${ }^{5}$ Cf. H. Miyazaki, $A$ note on paracompact spaces, Tôhoku Math. J. (2) vol. 4 (1952) pp. 88-92.

${ }^{6}$ Cf. J. Dieudonné, loc. cit. 
has property *, the natural quotient space of $X$ agrees with one constructed by Cech. ${ }^{7}$ Throughout the rest of this note, $D$ and $\phi$ always denote the natural quotient space and the projection for space $X$ provided $X$ has property *.

Lemma. Let $X$ be a space with property * and property **.

(3) Whenever $p \in D, \phi^{-1}(p)$ contains a smallest non-null closed set $F_{p} . F_{p}=\bar{x}$ for $x \in F_{p}$.

(4) Every open set which meets $F_{p}$ contains $\phi^{-1}(p)$. Hence for any $x \in X$, if $\bar{x}$ is covered by a system of open sets, $\bar{x}$ is contained in one of them.

(5) If $E, F$ are disjoint closed sets in $X$, then $\phi(E) \cap \phi(F)=\varnothing$.

Proof. Fix $p \in D$ and let $x_{0} \in \phi^{-1}(p)$. Clearly $\left\{\bar{x} \cap \bar{x}_{0}: x \in \phi^{-1}(p)\right\}$ is a system of closed sets in $\bar{x}_{0}$ and it has, by property ${ }^{*}$, the finite intersection property. It follows by property ** that

$$
F_{p}=\cap\left\{x: x \in \phi^{-1}(p)\right\}=\bigcap\left\{\bar{x} \cap \bar{x}_{0}: x \in \phi^{-1}(p)\right\}
$$

is a non-null closed set contained in $\phi^{-1}(p)$. For any non-null closed set $F$ contained in $\phi^{-1}(p)$, we have $F \supset \bar{x} \supset F_{p}$ whenever $x \in F$. Hence $F_{p}$ is the smallest. If $x \in F_{p}$, then $\bar{x} \subset F_{p} \subset \bar{x}$. Hence (3) is proved.

By construction $F_{p} \subset \bar{x}$ for each $x \in \phi^{-1}(p)$. Hence, if $U$ is open and meets $F_{p}, U$ meets $\{x\}$, i.e., $U$ contains each $x \in \phi^{-1}(p)$, proving that $\phi^{-1}(p) \subset U$. If $x \in X$ and $\bar{x}$ is covered by a system $\mathfrak{U}$ of open sets, then some open set of $\mathfrak{U}$ meets $F_{p}$ with $p=\phi(x)$, and hence contains $\bar{x}$. This proves (4).

If $E$ and $F$ are disjoint closed sets in $X$, then for $x \in E$ and $y \in F$, $\bar{x} \cap \bar{y} \subset E \cap F=\varnothing$ and so $\phi(x) \neq \phi(y)$. Hence $\phi(E) \cap \phi(F)=\varnothing$, proving (5).

Lemma. Let $X$ be a $T_{3}^{\prime}$ space with property **.

(6) $\phi$ is closed.

(7) Given any open covering $\left\{U_{\alpha}\right\}$ of $X,\left\{D-\phi\left(X-U_{\alpha}\right)\right\}$ is an open covering of $D$.

(8) $D$ is $T_{23}$ (i.e., $T_{2}$ and $T_{3}$ ).

(9) $D$ is $T_{24}$ (i.e., $T_{2}$ and $T_{4}$ ) or $L T_{24}$ (i.e., $T_{2}$ and $L T_{4}$ ) according as $X$ is $T_{4}$ or $L T_{4}$.

Proof. Let $F$ be a closed subset of $X$. Given any point $p$ of $D$ $-\phi(F)$ there is, by (3), a smallest non-null closed set $F_{p}$ contained in $\phi^{-1}(p)$. Take a point $x_{p}$ of $F_{p}$; there is, by $T_{3}^{\prime}$, a neighborhood $V_{p}$ of $x_{p}$ whose closure is contained in $X-F$. Therefore, by (4) and (5), $\phi^{-1}(p) \subset V_{p} \subset \bar{V}_{p} \subset X-\phi^{-1} \phi(F)=\phi^{-1}(D-\phi(F))$. Hence $\phi^{-1}(D-\phi(F))$

${ }^{7}$ Cf. E. Čech, On bicompact spaces, Ann. of Math. (2) vol. 38 (1937) pp. 823-844. 
$=U\left\{V_{p}: p \in D-\phi(F)\right\}$ is open and so $\phi(F)$ is closed. This proves (6). Given any open covering $\left\{U_{\alpha}\right\}$ of $X,\left\{D-\phi\left(X-U_{\alpha}\right)\right\}$ is a system of open sets in $D$ by (6). For each $p \in D$ there is, by (4), some $\alpha$ such that $\phi^{-1}(p) \subset U_{\alpha}$ and so $p \in D-\phi\left(X-U_{\alpha}\right)$. Hence (7) is proved.

By (6), $D$ is a $T_{1}$ space. Therefore $D$ is $T_{23}$ if we can show that for a point $p$ of $D$ and a neighborhood $G$ of $p$ there is a neighborhood of $p$ whose closure is contained in $G$. Take a point $x$ of $F_{p}$ and let $U$ be a neighborhood of $x$ with $\bar{U} \subset \phi^{-1}(G)$. Then $D-\phi(X-U)$ is a neighborhood of $p$ by (4) and (6) and its closure is contained in $G$ by (5).

Suppose that $X$ is $T_{4}$. Given any two disjoint closed subsets $P, Q$ of $D, \phi^{-1}(P)$ and $\phi^{-1}(Q)$ are disjoint closed subsets of $X$ and they have disjoint neighborhoods $U$ and $V$. Applying (6), we can easily see that $D-\phi(X-U)$ and $D-\phi(X-V)$ are disjoint neighborhoods of $P$ and $Q$, proving that $D$ is $T_{4}$ and hence $T_{24}$.

Suppose now that $X$ is $L T_{4}$. Let $p \in D$ and $x \in F_{p}$; there is, by hypothesis, a neighborhood $U$ of $x$ whose closure is normal. By (4) and (6), $G=D-\phi(X-U)$ is a neighborhood of $p$. Then there is, by $T_{3}$, a neighborhood $V$ of $p$ such that $\bar{V} \subset G$. Since $\phi^{-1}(\bar{V}) \subset \bar{U}$ is normal, it follows by the preceding result that $\bar{V}$ is normal, proving that $D$ is $L T_{4}$ and hence $L T_{24}$.

Lemma. Let $X$ be a pointwise paracompact (paracompact) space.

(10) $X$ has property**.

(11) If $X$ is $T_{3}^{\prime}$, then $D$ is pointwise paracompact (paracompact).

Proof. Fix a point $x_{0}$ of $X$. Given any system $\mathfrak{U}$ of open sets whose union contains $\bar{x}_{0}, \mathfrak{u} \cup\left\{X-\bar{x}_{0}\right\}$ is an open covering of $X$ and it admits a point-finite refinement $\mathfrak{B}$. Let $\mathfrak{B}^{\prime}=\left\{V: V \in \mathfrak{B}, V \cap \bar{x}_{0} \neq \varnothing\right\}$ $=\left\{V: V \in \mathfrak{B}, V \ni x_{0}\right\}$ and for each $V \in \mathfrak{B}^{\prime}$ we take a $U_{V} \in \mathfrak{U}$ such that $V \subset U_{V}$. Then $\mathfrak{U}^{\prime}=\left\{U_{V}: V \in \mathfrak{B}^{\prime}\right\}$ is a finite subsystem of $\mathfrak{u}$ which covers $\bar{x}_{0}$, proving the compactness of $\bar{x}_{0}$. Hence $X$ has property ${ }^{* *}$.

Given any open covering $\left\{G_{\alpha}\right\}$ of $D,\left\{\phi^{-1}\left(G_{\alpha}\right)\right\}$ is an open covering of $X$ and it admits, by hypothesis, a point-finite (locally finite) refinement $\left\{U_{\beta}\right\}$. By $(7),\left\{D-\phi\left(X-U_{\beta}\right)\right\}$ is an open covering of $D$ which is obviously point-finite (locally finite) and refines $\left\{G_{\alpha}\right\}$. Hence $D$ is pointwise paracompact (paracompact).

Theorem 2. A space $X$ is pointwise paracompact (paracompact) $T_{3}^{\prime}$ if and only if it has a pointwise paracompact (paracompact) $T_{23}$ retract $A$ which meets every non-null closed subset of $X$. Moreover, such a retract $A$ is $L T_{24}$ or $T_{24}$ if and only if $X$ is $L T_{4}$ or $T_{4}$. Finally, $A$ is unique up to a homeomorphism and the related retraction is uniquely determined. 
Proof. Suppose that $X$ is pointwise paracompact (paracompact) $T_{3}^{\prime}$. By (2), and (10), $X$ has property ${ }^{*}$ and property ${ }^{* *}$; it follows by (3) that for each $p \in D$ there is a smallest non-null closed set $F_{p}$ contained in $\phi^{-1}(p)$. We take, for each $p \in D$, a point $x_{p}$ of $F_{p}$ and denote by $A$ the set of these points $x_{p}$. Clearly $A$ meets every nonnull closed subset of $X$ and $\phi$ defines a 1-1 mapping $\psi$ of $A$ onto $D$. For any closed subset $F$ of $X$, we have $\psi(F \cap A)=\phi(F)$ which is closed by (6). Hence $\psi$ is a homeomorphism. From this result, we obtain a retraction $f=\psi^{-1} \phi$ of $X$ onto $A$. Moreover, it follows by (8) and (9) that $A$ is $T_{23}$ and that $A$ is $T_{24}$ or $L T_{4}$ according as $X$ is $T_{4}$ or $L T_{4}$.

Conversely suppose that $X$ has a pointwise paracompact (paracompact) $T_{23}$ retract $A$ which meets every non-null closed subset of $X$. Let $f$ be a retraction of $X$ into $A$. For any $x \in X, \bar{x} \cap A$ is non-null and $T_{2}$; therefore it contains exactly one point. From this result, it is easily seen that for any two points $x, y$ of $X, \bar{x} \cap \bar{y} \neq \varnothing$ if and only if $\bar{x} \cap A=\bar{y} \cap A$. Hence property * as well as property ** holds for $X$, and for each $p \in D, \phi^{-1}(p) \cap A$ contains exactly one point contained in $F_{p}$. From the latter result, we have, for $x \in X, f(x)=f(\bar{x})=\bar{x} \cap A$. Let $x \in X$ and let $U$ be a neighborhood of $\bar{x}$. Then there is a neighborhood $G$ of $\bar{x} \cap A$ in $A$ such that $\bar{x} \cap A \subset G \subset \bar{G} \cap A \subset U \cap A$. Hence $f^{-1}(G)$ is a neighborhood of $x$ whose closure is contained in $U$. This proves that $X$ is $T_{3}^{\prime}$.

If $A$ is $T_{4}$, then for any two disjoint closed subsets $E, F$ of $X$, $E \cap A$ and $F \cap A$ have disjoint neighborhoods $G$ and $H$ in $A$ and so $f^{-1}(G)$ and $f^{-1}(H)$ are disjoint neighborhoods of $E$ and $F$. Hence $X$ is also $T_{4}$. If $A$ is $L T_{4}$, then for any $x \in X, f(x)$ has a neighborhood $G$ in $A$ whose closure in $A$ is normal. It follows by the preceding result that $f^{-1}(G)$ is a neighborhood of $x$ whose closure is normal. Hence $X$ is also $L T_{4}$.

Given any open covering $\left\{U_{\alpha}\right\}$ of $X,\left\{U_{\alpha} \cap A\right\}$ is an open covering of $A$ and it admits, by hypothesis, a point-finite (locally finite) refinement $\left\{G_{\beta}\right\}$. We can easily see that $\left\{f^{-1}\left(G_{\beta}\right)\right\}$ is a point-finite (locally finite) refinement of $\left\{U_{\alpha}\right\}$. Hence $X$ is pointwise paracompact (paracompact).

According to the above argument, $A$ is homeomorphic to $D$ and hence is unique up to a homeomorphism. Moreover, for any $x \in X$, $\bar{x} \cap A=f(x)$. Hence the retraction $f$ is uniquely determined.

The author is much indebted to the referee for improving several results as well as for simplifying some proofs.

TULANE UNIVERSITY AND

UNIVERSITY OF ILLINOIS 